\title{
Transfer of Authority within Hierarchy ${ }^{1}$
}

\author{
Pinghan LIANG \\ Research Institute of Economics and Management \\ Southwestern University of Finance and Economics
}

August 28, 2010

\footnotetext{
${ }^{1}$ I'd like to thank the helpful comments from Alessandro Bonatti, Mikhail Drugov, Guillaume Haeringer, Oliver Hart, Sjaak Hurkens, Georg Kirchsteiger, David Martimort, Hannes Mueller, Nathanael Vellekoop, as well as the attendants in the workshops in UAB, Tilburg, ENTER Jamboree (2010), DMM (2010), ESNIE (2010), Stoney Brook Festival (2010), and 2nd Brazilian Workshop of Game Theory. E-mail address: liang.pinghan@gmail.com
} 


\begin{abstract}
Bureaucracy is featured by vertical hierarchical structure in which the decision maker usually lacks direct access to the informed agent, and the span of discretionary authority decreases top down. In this paper we investigate the delegation scheme in three-level hierarchies. The minister delegates authority to a biased senior bureaucrat, then the senior can make further delegation decision to the junior. We provide a full characterization of the implemented interval delegation set. It's shown that the efficiency is attained if and only if the senior is intermediately biased. We reverse the conclusion in Dessein (2002) that delegation dominates informative cheap talk and shows that, for some range of senior's preferences, the minister prefers keeping control and using the senior as information gate-keeper. The implication for hiring policy, job contents and promotion decision in bureaucracy is examined. Given the bias of the senior, the optimal junior should be intermediately between the senior and the minister. Under certain conditions the loyal bureaucrat would be assigned to action-level and doesn't get promotion. Complete delegation to an acquainted knowledgeable senior may arise endogenously by the minister's ignorance about the conflict of interest of the junior.

Key words: Delegation, Cheap Talk, Bureaucracy, Mediator, Hierarchy JEL classification codes: D72, D78, D82
\end{abstract}


The principles of office hierarchy and of levels of graded authority mean a firmly ordered system of super- and subordination in which there is a supervision of the lower offices by the higher ones.

Max Weber (1946, p. 214)

\section{Introduction}

In bureaucratic organization the top-level decision maker needs a chain of mediators (hierarchy) to deliver the commands and orders to the informed party. It's usually that neither can the expert communicates directly with the DM, nor may the DM command action-level subordinates. For example, in budget approval procedure the minister will set a ceiling on the budget within the senior bureaucrats' discretion, and the latter would further assign the decision-making right over a smaller amount to the junior officers. It's noteworthy that the span of discretionary authority is decreasing top down, and in many cases the minister is unable to skip the senior to authorize the juniors. In army, between divisions and battalions, regiments control information flow from below and pass commands from above. International organizations usually have to deliver the aid to local community via the authorization of national government. The question this paper addresses thus is: how to allocate decision-making right within hierarchy, and how to fill the positions of hierarchy?

To answer these questions, using the classical Crawford and Sobel (1982, henceforth, CS) cheap-talk model, we analyze organizational design in a three-layer minister/senior/junior hierarchies. All players want to adapt to the underlying true state, though they also gain different private benefit (bias). Only the junior agent will be informed about the true state. The minister chooses between delegating noncontractable decision right to the senior and keeping control rights when the junior communicates his information via the senior (mediator cheap talk). In other words, the minister (DM) has to control the junior (sender) via a strategic senior (mediator). We investigate the hierarchical delegation which refers to the situation that the minister not only assigns the control rights over a range to the senior, but also allows the latter to further delegate the decision right to the junior. Take the budget approval procedure as example, by delegation the minister imposes a budget cap on the senior, and then the senior decides whether to review the junior's proposal and make decision, or set a specified budget ceiling on the junior. By keeping control the minister hears the proposal of the junior through the senior and then allocates budget. 
We completely characterize the interval delegation set. The efficient delegation set obtained in minister-junior direct interaction (Holmstrom 1977, Alonso and Matouschek 2008, henceforth AM) is still implementable in this hierarchy if and only if the senior is intermediately biased, e.g., conservative senior bureaucrat and more conservative juniors. The intuition is that compared with the minister, the intermediately biased senior prefers to give more decision right to the junior, thus the minister can adjust it by imposing a binding cap on the delegation set to the senior. However, if the senior is opposite-biased (conservative senior vs. liberal junior) or more biased (more conservative senior vs. conservative junior), he strictly prefers assigning less discretion to the junior than the minister, which cannot be adjusted by the minister, so efficient delegation set is not implementable. Therefore a straightforward implication for the promotion decision is that if the minister can only choose whoever to be the senior, then the loyal bureaucrat should be promoted and given decision right over a limited set of action.

We compare the performance of delegation and communication in hierarchy and reverse the influential conclusion in Dessein (2002) that delegation dominates cheap talk whenever there exists an informative communication equilibrium $^{1}$. It's shown that when the senior and the junior are of opposite preferences, e.g., conservative-minded senior v.s. radical junior ${ }^{2}$, for some range of preference misalignment, delegation strictly underperforms cheap talk. The intuition is: on the one hand, compared with direct delegation, the gain from delegation reduces as the strategic senior becomes oppositebiased; on the other hand the opposite biased senior can improve communication efficiency by filtering information flow, i.e., using a specific garbling of information to relax incentive compatibility constraint (Myerson 1986), thus cheap talk with strategic senior can raise the minister's welfare relative to direct communication (Ivanov, 2009).

We then use the characterization of delegation to study the personnel management in bureaucracy, i.e., optimal selection of the senior and junior. We first study the situation that the minister can reorganize the department completely, namely the senior and the junior are chosen simultaneously. We reverse the previous results that the loyal agent should get promotion in that compared with appointing a more loyal agent to the senior position, this loyal person can benefit the minister more in the action-level junior position, so the minister gains in direct adaptation on the expense of indirect control. We then look at the selection of junior bureaucrat. It's shown that both the

\footnotetext{
${ }^{1}$ Dessein (2002) also investigates delegation through a mediator. However, he doesn't allow the mediator to further delegate, and only complete delegation is considered there.

${ }^{2}$ It could be that on a particular welfare program, the junior would like to increase expenditure while the senior is inclined to reduce expenditure.
} 
minister and the senior prefer to select the compromised agent as the actionlevel junior, i.e., the optimal perference of the junior would lie between the minister and the senior. Furthermore, we investigate the situation when the minister doesn't have the relevant knowledge about the preferences of the junior. The minister may optimally forgo the skip-level control of the unacquainted juniors, and grant an acquainted knowledgeable senior with full authority. Thus both the hierarchical structure of commands and orders and complete delegation can arise endogenously thanks to the asymmetric information between the minister and the senior.

The contribution of this chapter is two-fold. For the strand of mechanism design, following Renou and Tomala (2008), we show that network structure matters in choosing mechanism. The lack of direct access and the existence of strategic intermediaries may change the minister's choice between communication and delegation. For the research of organizational economics, our work contributes to the understanding of the Human Resource Management (HRM) practice in those government organizations which is characterized by strict hierarchy and the absence of contingent contract. We show that job design, hiring policy and promotion decision are interrelated ${ }^{3}$. Moreover, we provide an endogenous formation of hierarchy and complete delegation due to asymmetric information.

This work is organized as follows. In the following section we review the related literature. In Section 3 we lay out the basic model, and investigate the benchmark case of direct communication and delegation, which serves as the efficiency criterion. Section 4 characterizes the interval delegation set in hierarchy, Section 5 compares delegation with mediator cheap talk (communication). Section 6 studies the hiring policy of the junior and promotion decision, and investigate delegation scheme under uncertainty about bias. Section 7 discusses and concludes. All proofs are relegated in Appendix.

\section{Related Literature}

Based on CS model, GHPS (2009) establish that by hiring a neutral mediator the DM could implement the optimal mechanism to extract information from the informed expert. Ivanov (2009) and Ambrus et al (2009) show that this result is valid with a strategic mediator for some range of preferences (opposite bias). On the other hand, Dessein (2002) and AM (2008) analyze the delegation decision and demonstrate the dominance of delegation over cheap talk. These works more or less discuss delegation to a strategic uninformed mediator, but they didn't take into account the possibility that

\footnotetext{
${ }^{3}$ See Gibbons and Waldman (1999) for a accessible survey on this field.
} 
the mediator can further delegate to the informed party. Compared with these works, we allow further transfer of decision right and focus on optimal interval delegation scheme, instead of full delegation. We show that there exists some range of preference misalignment in which some communication outcome cannot be replicated by any delegation scheme. This new result has useful implication for understanding the allocation of authority in hierarchy.

A closely related paper is the recent work by Ambrus et al (2010), who compares the performance of closed rule and open rule in floor-committeelobbyist hierarchy. In terms of our work, open rule is equivalent to communication, while closed rule is complete delegation to the mediator (committee). They stress full delegation, and they don't allow the mediator (committee) to commit to any decision rule, e.g., rubberstamp the proposal of the lobbyist, even though the repeated interaction between the committee and the lobbyist may provide sufficient incentive for the former to stick to this decision rule. Thus our results on optimal interval delegation is more general. Moreover, both hierarchical delegation and complete delegation scheme can arise as endogenous outcome in our work.

There is a large body of literature in organizational economics which address the benefits of hierarchy. Largely motivated by bounded rationality, the works by Radner (1993), Bolton and Dewatripont (1994), and Geankoplos and Milgrom (1991) are concerned with the role of hierarchy in facilitating information processing, and stress the information aggregation within vertical structure. Based on heterogeneity among agents, another related strand of literature since Garicano (2000) develops "knowledge-based" hierarchy. As complementary, to a large extent we take hierarchy as a given structural characteristic, and address the strategic behavior of agents within it, analyze the selection of agents and choice between communication and delegation. It's noteworthy that our results that the hierarchy may arise as the result of the minister's uncertainty about the junior's personal interest is in accordance with the argument of limited information processing ability.

Tirole (1986) explicitly introduces supervisor into principal-agent relationship to study multiple-layer hierarchies. In his paper the supervisor holds private information about the type of agent, and the focus is the collusion between supervisor and agent. He establishes the equivalence between coalition-proof contract and giving ownership to a supervisor, who subcontracts with an agent further. We show that when monetary transfer is not feasible, which is common in many bureaucratic organization, the equivalence fails and subcontract cannot implement the optimal delegation scheme.

Our paper also contributes to the growing body of literature on strategic communication embodied in network structure (Renou and Tomala, 2008, Calvo-Armengol et al, 2009). In particular, in the sense that the implemen- 
tation of optimal mechanism in network structure is addressed, we are closely related to Renou and Tomala. However, we differ from this line of research in highlighting a specific mechanism: the allocation of authority. We add a possibility that network structure may affect the decision of mechanism designer, thus mechanism choice is affected by the network.

\section{Model}

An organization is composed of three players: a minister ("she", denote as player $D($ ecision $) M(a$ ker $))$, a senior bureaucrat, and a junior bureaucrat ("he", denote as player $s b$ and $j b$, respectively). The utility of each player is of quadratic form as the classical CS model:

$$
U\left(\theta, y, b_{i}\right)=-\left(\theta-y+b_{i}\right)^{2}, i=D M, s b, j b
$$

Thus their payoffs depend on the true state $\theta \in \Theta=[0,1]$, the action undertaken $y \in Y=\mathbb{R}$, and their private benefits $b_{i}$. Each player wants to adapt to the true state though to different extent, i.e., the ideal action is $\theta+b_{i}$. Without loss of generality, we normalize $b_{D M}=0$ and use $b_{s b}, b_{j b}$ to measure the discrepancy of interest between officers and the minister, e.g., the bureaucrats may want to exaggerate the budget to enlarge his subordination. For the sake of simplificity, we use $U_{i}(\theta, y)$ to refer the utility of player $i$.

The minister has the right to take action, and only the junior would be informed about the true state $\theta$, but he could not communicate directly with the minister, neither the minister can allocate the authority directly to the junior. In other words, the senior has full control of the information transmission between the minister and the junior. The senior and the minister have uniform prior on $\Theta$.

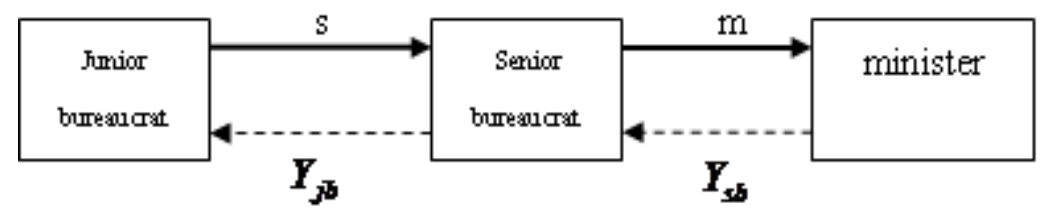

Figure 1 Timeline under communication and delegation (The solid and dashed line represents communication and delegation, respectively)

Now we specify the timing of this game under delegation and communication in Figure 1. The minister first assigns two agents to the senior and the junior positions, and only the junior learns the true state. If the minister engages in communication, as the solid line in Figure 1, the junior first 
delivers message from the signal space $S$ to the senior, and the senior in turn chooses $m$ from the message space $M$ and send to the minister. The strategy for the junior thus is $\mu_{j b}: \Theta \rightarrow \Delta S$, and for the senior is $\mu_{m}: S \rightarrow \Delta M$. The minister forms a posterior about the true state conditional on the message received from the senior, and chooses his ideal action $y: M \rightarrow Y$. The optimal response thus is $y=E[\theta \mid M]$. To get rid of multiple equilibria problem common in cheap talk game ${ }^{4}$, we would focus on the most informative equilibrium, i.e., the Pareto-dominance one.

Alternatively, if the minister chooses delegation, she gives the senior full control over the interval $Y_{s b} \subset Y$, e.g., the amount of budget he can choose. The senior can ask the junior to send message from the signal space $S$, and implement his best response $y_{s b}=\arg \max -\left(E[\theta \mid S]-y+b_{s b}\right)^{2}$. Otherwise, the senior can subcontract, i.e., delegate decision right, with the junior by granting him the list of actions $Y_{j b} \subset Y_{s b}$, as the dashed line in Figure 1.

\subsection{Benchmark: direct interaction}

We start from the benchmark case that the sender can send messages to the decision maker directly, who then updates belief about the true state and makes decision. Thus we return to the classical CS model, as Lemma 1 summarizes

Lemma 1 (CS 1982) If the informed party could talk with the decision maker directly, then informative communication occurs if $\left|b_{j b}\right| \leq \frac{1}{4}$, and in equilibrium the induced set of actions consists of finite elements.

This pessimistic result obtains due to the decision maker's inability to commit, which means that the DM would update his belief and select ex post best action upon receiving message. The DM gains by keep the control but loses in motivating the sender to provide information. The information loss is significant since only finite actions would be undertaken. The communication is informative if the minister would have finer partition about the state after hearing messages. It naturally leads to questioning whether and how the DM could improve the efficiency.

By delegating authority to the informed party, the DM (minister) allows the agent to implement his preferred action from a prescribed set, thus this is in effect equivalent to making commitment to a decision rule. The DM forges

\footnotetext{
${ }^{4}$ Babbling equilibrium (Uninformative communication equilibrium) always exists in cheap talk game, in which the DM would always implement the ex ante optimal action $E[\theta]=\frac{1}{2}$
} 
the flexibility in decision-making to facilitate the use of information. In particular, there is full information transmission under complete delegation in that the preferred action of informed party could be always selected. Goltsman et al (2009, henceforth GHPS) establishes that the optimal delegation scheme a la Holmstrom (1977), in which the informed party is given control over a limited interval of $Y$, can attain the second-best optimal outcome whenever the DM has commitment power.

Lemma 2 If the decision maker can delegate authority to the informed party, then outcome would be better than optimal communication outcome if $0 \leq$ $b_{j b} \leq \frac{1}{2}$. The optimal delegation set is $Y^{*}=\left[0,1-b_{j b}\right]$, and the action chosen is

$$
y(\theta)=\left\{\begin{array}{c}
\theta+b_{j b}, \text { if } \theta \in\left[0,1-2 b_{j b}\right] \\
1-b_{j b}, \text { otherwise }
\end{array}\right.
$$

The optimal delegation set of action is an interval truncated on the top, which is determined by the intensity of conflict of interest between the minister and the informed party. Optimal delegation seeks the balance between the loss of control right and the gain of information. In low state the latter effect outweighs, thus the informed party is allowed to act according to his interest. The former effect dominates in high state and the decision maker keeps de facto control by imposing a ceiling $\left(1-b_{j b}\right)$. Thus the information is perfectly revealed in the lower interval, whose size is decreasing with respect to the preference misalignment. This optimal delegation would serve as the efficient delegation set for our analysis on hierarchical delegation. The expected utility for the minister under this second-best thus is:

$$
E U_{D M}=-\int_{0}^{1-2 b_{j b}} b_{j b}^{2} d \theta-\int_{1-2 b_{j b}}^{1}\left[\theta-\left(1-b_{j b}\right)\right]^{2} d \theta=\left(\frac{4}{3} b_{j b}-1\right) b_{j b}^{2}
$$

Various authors have provided characterization of optimal delegation mechanisms, including using arbitration to resolve dispute (Melumad and Shibano, 1991), optimal interval delegation (AM 2008), veto-power of the decision maker (Mylovanov, 2008), and stochastic delegation (Kovac and Mylovanov, 2009). More precisely, AM establishes the conditions for the interval delegation, which is restated in current environment as the following:

Lemma 3 The optimal delegation set from the minister to informed agent is a connected set. 
This lemma states that the delegation set contains either no decision, one decision $^{5}$ or an interval of decisions. For negatively biased junior we have that the optimal delegation set is truncated on the bottom.

Corollary 1 If $-\frac{1}{2} \leq b_{j b}<0$, then efficient delegation set is $Y^{*}=\left[-b_{j b}, 1\right]$.

This corollary is a straightforward application of Lemma 2. Since now the junior bureaucrat is downwardly biased, the consequence of conflict of interest is more intense in low state, thus the minister needs to restrict the discretion of the junior there. Therefore, now the lower bound is present instead of the cap.

\section{Hierarchical Delegation}

Under interval hierarchical delegation, the minister cannot directly contact with the action-level junior bureaucrats ${ }^{6}$. Because it has already established that meaningful delegation, which means that there are more than one action in delegation set, requires that $b_{j b} \leq \frac{1}{2}$. We would focus on the case that $\left|b_{s b}\right|,\left|b_{j b}\right| \leq \frac{1}{2}$.

We would use $\Delta \equiv b_{j b}-b_{s b}$ to represent the divergence of conflict between the junior and the senior. As a useful benchmark, we first examine the situation that the delegation is not allowed to transfer. In other words, the senior is allowed to select action from a set of alternatives, but he is not allowed, or lack the necessary commitment power, to further delegate this authority to the junior (Dessein 2002, Ambrus et al, 2010). Therefore the standard CS cheap talk applies between them, and the results are similar to Lemma 1 , though now $\Delta$, rather than $b_{i}$, determines the equilibrium outcome, and the final decision undertaken may be restricted by the minister.

Then we check the performance when subcontract (further delegation to the junior) is possible. We say an interval of actions $Y^{\prime}$ is implementable if $Y^{\prime} \subseteq Y_{j b} \subseteq Y_{s b}$, in other words, the actions realized should be in the interest of the junior, the senior and the minister. The implemented set is the final delegation interval of the junior in the sense that only the junior has the relevant expertise to choose proper action. The senior acts as if a

\footnotetext{
${ }^{5}$ This would be the ex ante optimal response $E_{D M}[\theta]=\frac{1}{2}$.

${ }^{6}$ The senior bureaucrats may actively prevent the direct link between his subordination and supervision. The behavior of Sir Humphrey Appleby, the permanent undersecretary in the fictional Department of Administrative Affairs in the BBC series Yes, Minister, vividly illuminates this point.
} 
mechanism designer whose set of available actions is restricted to an interval $Y_{s b}$, and makes delegation decision based on the relative difference of bias $\Delta$, thus the only way of the minister to control delegation is to restrict $Y_{s b}$. The delegation rule available to the minister thus is subject to the incentive compatibility of senior.

Lemma 3 immediately leads to that if $Y_{s b}$ is an interval or a point, so is $Y_{j b}$. The minister thus will just limit the menu of actions by imposing upperand lower-bound. Then we turn to characterize the implemented delegation set of the junior.

Proposition 1 If $\Delta>0$, the highest available alternative to the junior $\overline{y_{j b}}=$ $\min \left\{1-\Delta+b_{s b}, \sup \left\{y \mid y \in Y_{s b}\right\}\right\}$

This proposition demonstrates the impact of conflict of interest between the minister and the senior. If the senior is granted with large discretion power, then he would act in his own interest, and the delegation set implemented $\left(\left[0,1-\Delta+b_{s b}\right]\right)$ differs from the efficient one $\left(\left[0,1-b_{j b}\right]\right)$, as shown in Figure 2. However, it also suggests that the minister could control the loss from this divergence of ideal actions by truncating the delegation set, i.e., imposing upper-bound $1-b_{j b}$ on $Y_{s b}$. The efficient delegation set would be implemented in hierarchical structure.

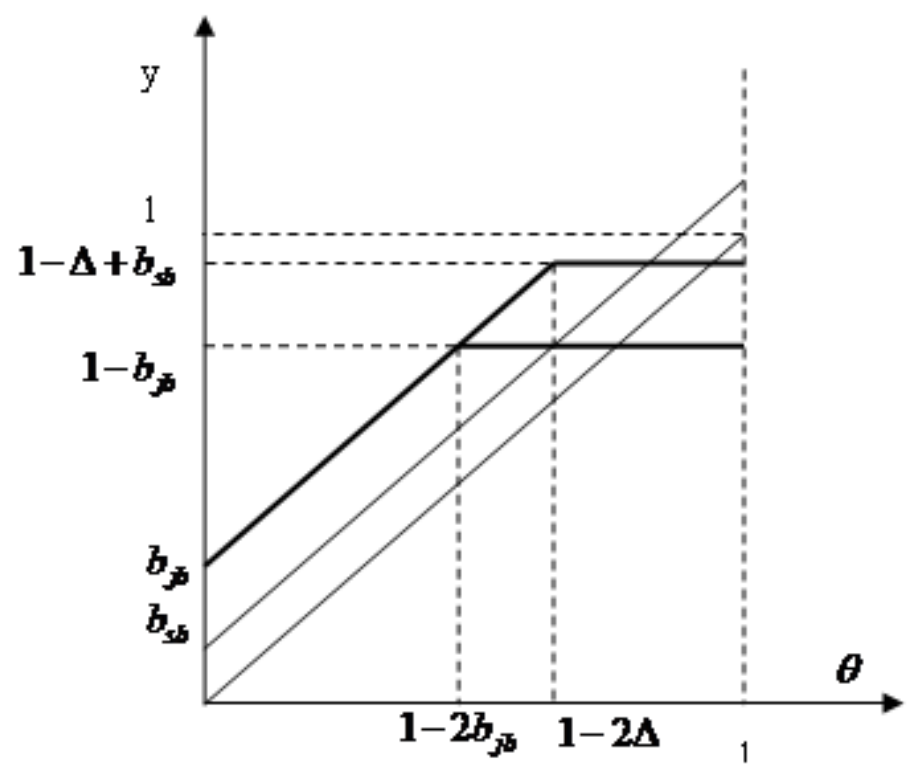

Figure 2. Property of optimal delegation set from DM and the mediator standpoint, respectively. The bold line depicts the action chosen as function of state $\theta$. 
Corollary 2 If the two bureaucrats are both positive biased and the senior is less biased, i.e., $b_{j b}>b_{s b}>0$, then the efficient delegation set $Y^{*}$ is implemented by imposing $\sup \left\{y \mid y \in Y_{s b}\right\}=1-b_{j b}$

It's routine to check that if these two agents are negatively biased and $b_{j b}<b_{s b}$, then the efficient delegation set is still implementable, though now the device to get it is to truncate delegation set $Y_{s b}$ at the bottom.

Therefore, even though the senior is biased, the minister still can replicate her optimal outcome under direct interaction by appointing a less biased senior and truncating the delegation set. This intermediately biased senior would like to widen the discretion of the junior, but this conflict of interest can be controlled by the minister by imposing a tight ceiling. The expected utility of the minister thus would be the one in (2). Obviously, it significantly increases the payoff to the minister relative to the case that the authority is not allowed to be transferred. The implication to promotion decision thus is given the conflict of interest among the bottom-level bureaucrats, the minister should fill the undersecretary position with the person whose preference is aligned, e.g. more "loyal" or "like-minded". The prediction that the minister would like to assign the loyal agent to important position also receives some empirical support from Iyer and Mani (2009), who find out that in India the elected politician (Chief Minister) will award the loyal professional bureaucrats with important positions.

However, this results crucially depends on that the senior is intermediately biased. If the senior is more biased, or two agents are of opposite direction of bias, then compared with the minister, the senior strictly prefers assigning less discretion to the junior, and this implementation of efficient outcome fails.

Proposition 2 If the two bureaucrats are opposite biased or the senior is more biased than the junior, then the efficient delegation set would never be implementable, i.e., $Y_{j b} \neq Y^{*}$.

We illustrate the failure of implementable efficient delegation set in the following figures. In Figure 3(a), when two agents are of opposite direction of bias, the elements of efficient delegation set are everywhere higher than those in $Y_{j b}$, so $Y_{j b} \subset Y^{*}$ if $Y^{*} \subset Y_{s b}$. Thus the minister has no way to force the senior to authorize the junior to take any higher action than $1-\Delta$. In particular, for meaningful delegation we need these two agents are not too extremely biased when they have opposite interest, namely $\Delta<\frac{1}{2}$. On the other hand, as shown in Figure 3(b) when the senior is more biased, though the minister prefers to truncate the junior's behavior in high state, the senior 
is inclined to restrict the junior's discretion in low state. Thus any action lower than $-\Delta$ would not be authorized by the senior. Thus the minister and the senior differ in the direction in controlling the informed party.

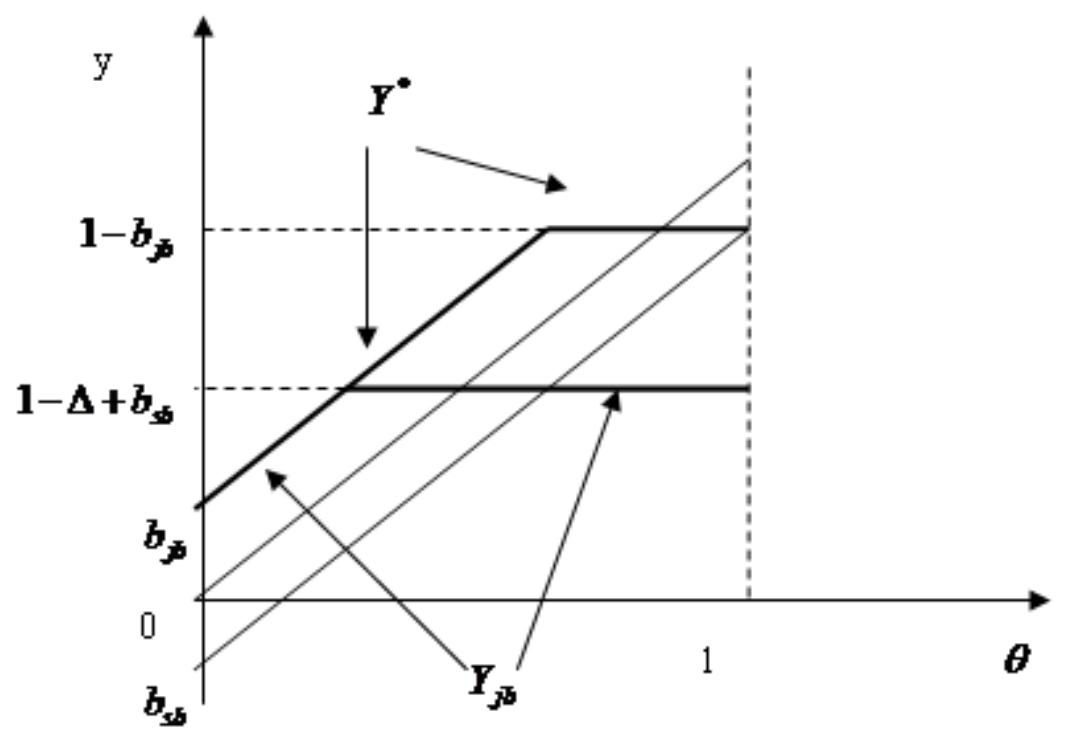

Figure 3(a). The delegation set with opposite biased agents

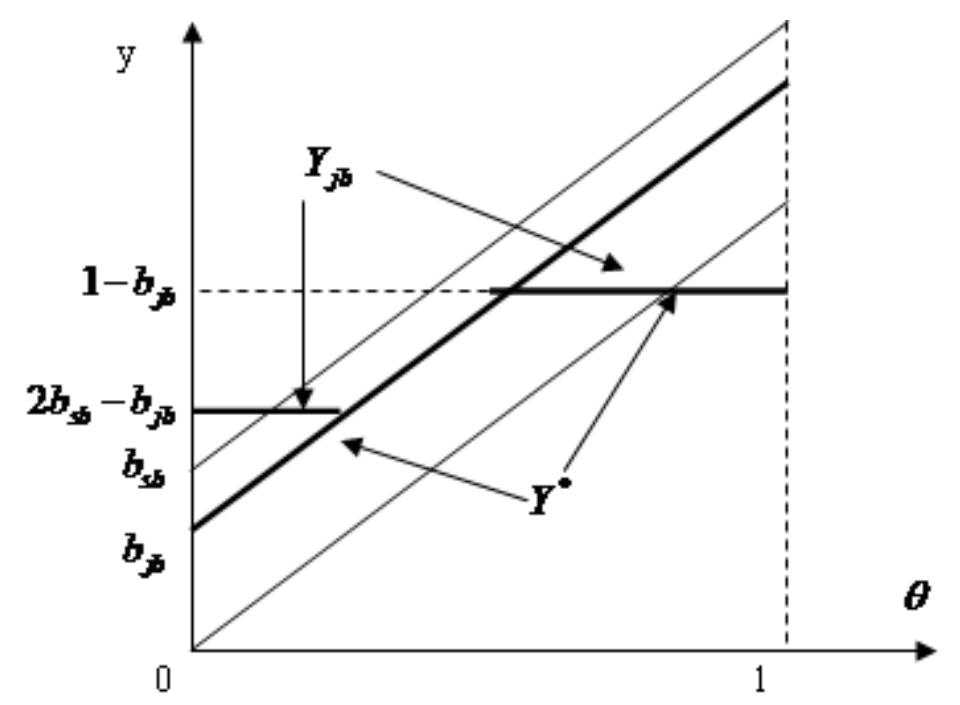

Figure 3(b). The delegation set with extremely biased mediator

Finally, we fully characterize the optimal implemented delegation set under different bias of agents and the expected payoff to the minister. We should keep in mind that when either $|\Delta| \leq 1 / 2$ or $\left|b_{i}\right| \leq 1 / 2$ fail, the minister prefers to take ex ante optimal action $\frac{1}{2}$ and there is no information gain 
from delegation, the expected payoff thus would be $-\frac{1}{12}$. To focus on the issue of interest and for the sake of simplicity, we assume that $\frac{1}{2} \geq b_{j b} \geq 0$ (the case of $b_{j b}<0$ is perfectly symmetric), and there is no limitation on $b_{s b}$.

Theorem 1 The optimal implemented delegation set prescribes the alternatives available to the informed junior. Formally, it satisfies:

$$
Y_{j b}=\left\{\begin{array}{cc}
{\left[0,1-b_{j b}\right],} & \text { if } \frac{1}{2} \geq b_{j b}>b_{s b} \geq 0 \\
{\left[0,1+2 b_{s b}-b_{j b}\right],} & \text { if } b_{j b}>0>b_{s b} \text { and } \Delta \in\left[0, \frac{1}{2}\right] \\
{\left[2 b_{s b}-b_{j b}, 1-b_{j b}\right],} & \text { if } b_{s b}>b_{j b}>0 \text { and }|\Delta| \in\left[0, \frac{1}{2}\right] \\
\frac{1}{2}, & \text { otherwise }
\end{array}\right.
$$

and the minister's expected utility is

$$
E U_{D M}^{H D}=\left\{\begin{array}{cc}
\left(\frac{4}{3} b_{j b}-1\right) b_{j b}^{2}, & \text { if } \frac{1}{2} \geq b_{j b}>b_{s b} \geq 0 \\
-b_{j b}^{2}+\frac{8}{3} b_{s b}^{3}-4 b_{s b}^{2} b_{j b}+\frac{4}{3} b_{j b}^{3}, \text { if } b_{j b}>0>b_{s b} \text { and } \Delta \in\left[0, \frac{1}{2}\right] \\
-\frac{8}{3} b_{s b}^{3}+4 b_{j b} b_{s b}^{2}-b_{j b}^{2}, \quad \text { if } b_{s b}>b_{j b}>0 \text { and }|\Delta| \in\left[0, \frac{1}{2}\right] \\
-\frac{1}{12}, & \text { otherwise }
\end{array}\right.
$$

The expected payoff to the senior officer is

$$
E U_{s b}^{H D}=\left\{\begin{array}{cc}
-\Delta^{2}\left(1-2 b_{j b}\right)-\frac{2 b_{j b}}{3}\left(b_{j b}^{2}+3 b_{s b}^{2}\right), & \text { if } \frac{1}{2} \geq b_{j b}>b_{s b} \geq 0 \\
-\Delta^{2}+\frac{4}{3} \Delta^{3}, \quad \text { if } b_{j b}>0>b_{s b} & \text { and } \Delta \in\left[0, \frac{1}{2}\right] \\
\frac{\Delta^{3}}{3}-\Delta^{2}\left(1-2 b_{s b}\right)-\frac{\left(b_{j b}+b_{s b}\right)^{3}}{3}, & \text { if } b_{s b}>b_{j b}>0 \text { and }|\Delta| \in\left[0, \frac{1}{2}\right] \\
-\frac{1}{12}-b_{s b}^{2}, & \text { otherwise }
\end{array}\right.
$$

This theorem shows that the discrepancy of interest of the senior is irrelevant to the implementation of optimal delegation scheme as long as it lies between the minister and the junior. Otherwise, the span of discretionary authority of the junior, i.e., the implemented delegation set, would be increasing (decreasing) with respect to the bias of the senior if it's negative (positive). Moreover, $\frac{\partial E U_{D M}^{H D}}{\partial b_{s b}}<0$ if $b_{s b}>b_{j b}$, and $\frac{\partial E U_{D M}^{H D}}{\partial b_{s b}}>0$ if $b_{s b}<0$. Thus in general the more loyal the senior to the minister, the larger gain in delegation. 


\section{Comparison with Hierarchical Communi- cation}

We have established the relationship between the performance of delegation and the preference of bureaucrats, and shown that it's ideal for the minister to appoint a loyal senior. In reality, however, usually the selection of the officers is not made by the direct supervisor. For example, in the U.S. system of separate powers, the executive (the President) appoints the administrative agency managers, e.g., FDA, while the Congress dictates policy and oversees its implementation. In terms of our model, the decision maker (Congress) can choose neither the mediator (FDA) nor the informed sender (the pharmaceutical company). Alternatively, she would respond by varying the level of oversights (Warren, 2008).

In this section we ask the specific question: if the minister cannot select any bureaucrats, under what condition the delegation scheme will be chosen? In particular, we compare hierarchical delegation with hierarchical communication given the preference of bureaucrats. This investigation involves the allocation of authority in hierarchy: should the senior be given some decision making right, or just act as an information gate-keeper? We find out that the inability to access the informed party may reverse the conclusion in previous study that the DM is better off by delegating.

Hierarchical communication shares with hierarchical delegation with the same structure: vertical hierarchy, but differs in whether the minister relegates some controls to the senior. Under hierarchical communication in equilibrium the senior can only convince the minister on which partition the state lies in, i.e., the minister takes decision as the optimal response to a certain partition.

The uninformed mediator can improve information transmission upon direct cheap talk by using mixed strategy equilibrium, i.e., mix or conceal some messages (Mitusch and Strausz, 2005). If the senior prefers less expenditure on a specific program than the minister, while the junior wants more budget, then upon hearing from the junior, it"s optimal for the senior to distort the message downward randomly. Anticipating this random distortion, the junior's incentive of exaggregation reduces. In particular, he realizes that the final outcome is not even monotonic with respect to his reports. Therefore he would like to speak out more honestly ${ }^{7}$. The minister thus gains by relaxing incentive compatibility constraint of the junior. Ivanov (2010) shows that

\footnotetext{
${ }^{7}$ The intuition is also explored by Blume et al (2007), in which noise in communication invalids the monotonicity condition of action with respect to message.
} 
with a properly biased mediator, the DM could attain the optimal cheap talk outcome a la GHPS (2009).

Lemma 4 (Ivanov 2009) For any $b_{j b} \in\left[0, \frac{1}{2}\right]$, there exists a mediator with bias $b_{s b} \in\left(-2 b_{j b}, 0\right]$ and an equilibrium in the game with this mediator that provides $E U_{D M}^{H C}=-\frac{1}{3} b_{j b}\left(1-b_{j b}\right)$. However, if $b_{s b} \in\left[0, b_{j b}\right)$, the mediator communication could not improve upon direct talk.

The intermediately biased senior $\left(0<b_{s b}<b_{j b}\right)$ could not improve efficiency upon direct talk since mixing messages is not in his interest. We could compare the maximum efficiency in communication $E U_{D M}^{H C}$ with the outcome of hierarchical delegation. Since $\frac{\partial E U_{P}^{H D}}{\partial b_{s b}} \geq 0$ when $b_{s b}<0$, if there is a $b_{s b}^{\prime}$ such that $E U_{D M}^{H D}\left(b_{s b}^{\prime}\right)=-\frac{1}{3} b_{j b}\left(1-b_{j b}\right)$, we could say that for any $b_{s b}>b_{s b}^{\prime}$, hierarchical delegation strongly dominates mediator cheap talk in the sense it can achieve higher payoff than the maximal payoff attainable in mediator talk. The resulted $b_{s b}^{\prime}$ turns out to be a nonlinear function of $b_{j b}$, which is formally defined by the following equation:

$$
-b_{j b}^{2}\left(1+2 b_{s b}^{\prime}\right)+\frac{\left(2 b_{s b}^{\prime}-b_{j b}\right)^{3}}{3}+\frac{5 b_{j b}^{3}}{3}=-\frac{1}{3} b_{j b}\left(1-b_{j b}\right)
$$

The higher dashed curve OB in Figure 4 describes this indifference line.

Unfortunately, there is non-monotonic relationship between the existence $k$-action mixed equilibrium and the bias of senior. Ambrus et al (2009) show that though two-action mixed equilibria requires that $b_{s b}$ is "sufficiently" far below zero, 3 -action mixed equilibria can exist when both $b_{j b}$ and $b_{s b}$ are close to 0 . This non-monotonicity in hierarchical communication limits complete characterization about the relative efficiency of two organization modes.

However, we still obtain the striking finding that informative communication may dominates delegation. To illustrate this, we compare the range of values of $b_{s b}$ for the existence of two-interval equilibrium in $H C$ mode and meaningful $H D$ mode (the minister would find out optimal to delegate more than one decision). We relegate in Appendix B the construction of two-action mixed equilibria under $H C$, and note that any meaningful $H D$ requires the following inequality being satisfied:

$$
-b_{j b}^{2}\left(1+2 b_{s b}\right)+\frac{\left(2 b_{s b}-b_{j b}\right)^{3}}{3}+\frac{5 b_{j b}^{3}}{3} \geq-\frac{1}{12}
$$


Namely for the minister delegating more than one decision is better than taking ex ante optimal action $\frac{1}{2}$.

Figure 4 demonstrates this comparison. OB and FB represents (6) and (7), respectively. Thus the delegation is meaningful only if $b_{s b}$ lies above FB. The trapezium ABCD specifies the range of values of $b_{s b}$ for which the twopartition equilibrium exists ${ }^{8}$. For slightly biased senior $\left(b_{s b}\right.$ higher than the higher dashed curve OB), delegation strictly dominates mediator cheap talk in the sense that it generates higher expected payoff than the best attainable under communication. For large biased senior $\left(b_{s b} \leq-\frac{1}{4}\right.$ but $\left.\Delta \leq \frac{1}{2}\right)$ and less biased junior (the lower triangle DEF), again delegation dominates. However, when the junior becomes more biased and the senior is only modestly biased, like those $b_{s b}$ lies in the shadow triangle BCE, communication would be informative and dominates any delegation scheme.

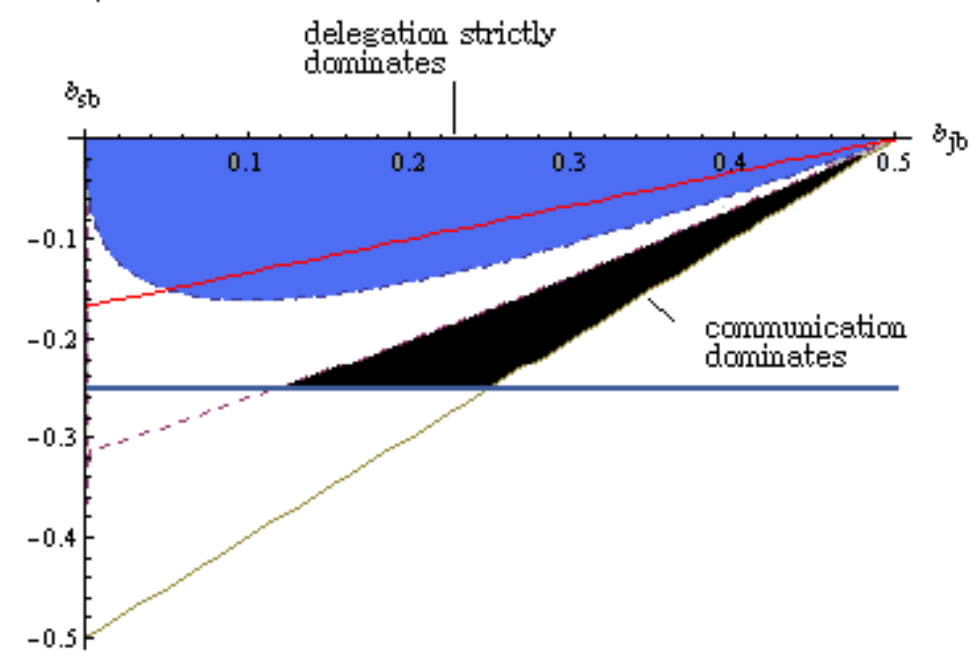

Figure 4. The comparison of expected payoff under $H D$ and $H C$ modes.

Therefore, if the two officers are of same direction of bias and given hierarchy, i.e., both conservative, then the minister should delegate the senior with limited discretion power. On the other hand, if the two bureaucrats are of opposite interest, i.e., conservative senior vs. radical liberal junior, in particular if the senior is slightly conservative but the junior is very radical, it's possible that communication could induce informative talk but delegation is trivial in that only one decision is relegated. The minister may keep control rights and restrict the role of the undersecretary to information processing, instead of delegate decision rights. The following numerical example exemplifies this claim:

\footnotetext{
${ }^{8}$ It's defined by $0 \geq b_{s b} \geq-\frac{1}{4}$ and $b_{j b}-\frac{1}{2} \leq b_{s b} \leq \frac{b_{j b}}{3}-\frac{1}{6}$.
} 
Example 1 Suppose $b_{j b}=\frac{1}{3}$, the upper-bound of communication efficiency $i s-\frac{2}{27}$, which could be achieved by choosing $b_{s b}=-\frac{1}{9}$. On the other hand, by $(6)$, if $b_{s b} \geq-0.089$, then delegation strongly dominates communication. Hence, for some range of the senior's bias, for instance, $b_{s b}=-\frac{1}{9}$, communication generates higher expected payoff than delegation.

Therefore we reverse the central conclusion in Dessein (2002) regarding the efficiency of delegation, in which whenever there is informative communication in cheap talk, delegation dominates communication. Moreover, as GHPS have shown, under direct interaction the limited delegation scheme can implement the second-best outcome where the DM has commitment power. Thus by optimal delegation the minister could do no worse than cheap talk. Hence the minister should always engage in delegation instead of communication. However, we show that this result would not hold when the minister cannot access informed party. It's possible that for some range of bias there is gain in mediator talk but no meaningful delegation scheme.

Because the minister cannot access the junior, i.e., she cannot observe message $s$, she has to take the incentive of senior into account when making decision based on $m$. Moreover, any decision rule she commits to has to be in the best interest of senior. In other words, due to the fact that the senior controls information flow, there are only limited action rules available to the minister under delegation. When the minister finds out that under any available decision rules the loss of control is too large she may forgo delegation and engage in communication, in which the gain in keeping decision right outweighs the loss in information transmission. The minister is reluctant to delegate or rubberstamp not due to commitment cost or ex post temptation to renege, as previous works suggest. Instead, it's the inability to interact with the informed party directly. As consequence, the possible solution to this problem is not offering commitment device or reputation concerns. Instead, organization design, in particular the layers in organization, matters in solving commitment problem and providing proper incentives for real informed agent.

This result also suggests that job design is related to hiring policy since the loyalty of senior determines what the senior could do. The elected politician would like to retain control over decision if she cannot replace the bureaucrats. Thus even though the hierarchy as a structural feature of bureaucracy is well defined, the job content of each position depends on the authority of the minister over personnel management. 


\section{Personnel Management in Hierarchy}

In this section we would investigate the personnel management in bureaucratic organization, i.e., how to fill the positions in hierarchy. This involving question contains the choice of senior and junior, i.e., the hiring policy and promotion decision.

The first question is given the conflict of interest of two officers, if the minister can reorganize the department, which one should be assigned to higher position? It differs from promotion decision in that it involves complete reassignment of jobs, instead of promote someone while leave the junior level unchanged. To deal with this question, we assume that both bureaucrats are of positive bias, i.e., $b_{2}>b_{1}>0$. The minister thus needs to tradeoff between using the more biased junior 2, in which the information loss only occurs on the top, and using the less biased junior 1, where the minister may gain information in high state on the expense of loss in low state. We find out that the more biased agent will be assigned to the junior position only if his bias is sufficiently far from the other agent.

Corollary 3 The less biased officer 1 will be assigned to the junior position if $b_{2}<\frac{1+\sqrt{1+16 b_{1}}}{8}$.

Therefore, a moderately biased senior can help controlling the more biased junior, but the minister can also find out that it may be beneficial to assign this moderately biased agent to the action-level and promote the more biased guy, in order to enlarge the range of implemented delegation set. While a loyal senior provides the minister with better control of the informed party indirectly, the loyalty in the action level reduces the loss of control directly. The indirect gain in control due to loyal senior cannot compensate the direct loss if the other candidate is not very biased. Thus given the pool of bureaucrats, it's not necessarily that the senior is more loyal, even if the minister has the full power to organize the hierarchy and assign the jobs.

\subsection{Selecting the junior}

We have studied the choice of the senior for the minister, given the bias of the junior. It corresponds to the promotion policy in bureaucratic organization, and we show that the more loyal bureaucrat should be placed into senior position.

In reality, however, usually the minister cannot remove the senior officer. For example, in UK government the permanent undersecretary is the non-political civil service head of a government department, they report and 
advise the Secretary of State, and are answerable to Parliament. However, the minister may have some voices in screening the entry-level (junior) bureaucrat. In this subsection we explore the selection of the junior given the preference of the senior and the minister.

We undertake some comparative statics about the expected payoff of the minister. First, $\frac{\partial E U_{D M}^{H D}}{\partial b_{j b}} \leq 0$ for almost all cases ${ }^{9}$, which means that the minister is harmed by the conflict of interest between the minister and the informed junior. Second, there exists complementarity between the bias of the junior and the senior $\left(\frac{\partial E U_{D M}^{H D}}{\partial b_{j b} \partial b_{s b}} \geq 0\right)$.

To understand the implications of these comparative statics, we should think about the hiring policy of bureaucratic organization. Given the preference of the senior officer and a pool of candidates for the junior position, which one should be selected as the junior bureaucrat? Suppose the senior is conservative $\left(b_{s b}>0\right)$, then if the neutral minister chooses a more conservative junior $\left(b_{j b}>b_{s b}\right)$, then the efficient delegation set is implementable (the first part of (3)), and the expected payoff of the minister (the first part of (4)) is decreasing with respect to the conflict of interest of the junior. Therefore, the minister would like to appoint a bureaucrat no more conservative than the senior. On the other hand, if the minister selects a liberal junior $\left(b_{j b}<0\right)$, then the senior wants to impose more restriction on the discretionary of the junior than the minister, thus the minister again gains by appointing a less liberal junior.

Hence to check the increasingly conservative senior bureaucrat, the minister should either select the less radical one if all candidates are liberal, or choose optimally conservative one ${ }^{10}$ if all just differ in the degree of conservatism. But in general, the minister would like to appoint a compromise junior $\left(b_{s b}>b_{j b}>0\right)$. The general implication is the minister will check the conflict of interest of the senior by deliberating selecting the junior. Iyer and Mani (2009) also examine the Chief Minister/District Politician/Bureaucrat hierarchy in India, and find out that when the Chief Minister and the District Politician are elected from the different parties, then the Chief Minister is more likely to change bureaucrat. In broad sense our implication is consistent with their results.

More interesting results can be obtained if the minister (DM) and the senior (mediator) have joint control over the selection of junior (sender). To focus on issue of interest, we assume that $b_{j b}, b_{s b} \in\left(0, \frac{1}{2}\right)$, i.e., the bureaucrats

\footnotetext{
${ }^{9}$ If $b_{s b}>b_{j b}>0$, then $\frac{\partial E U_{D M}^{H D}}{\partial b_{j b}} \leq 0 \Leftrightarrow b_{s b}^{2} \leq \frac{b_{j b}}{2}$. If $b_{s b}<0<b_{j b}$, then $\frac{\partial E U_{D M}^{H D}}{\partial b_{j b}} \leq 0$ holds for any $b_{j b} \in\left[0, \frac{1}{2}\right]$.

${ }^{10}$ As we derive in footnote 9 , it should be $b_{j b}=2 b_{s b}^{2}$ if $b_{s b} \leq \frac{1}{2}$
} 
have the same direction of bias, like conservatism, but differ in the extent. Therefore, the selection of junior will depend on the total expected welfare of the minister and the senior.

Suppose the expected payoff of the minister and the senior enter the total welfare equally. By adding (4) and (5) together we will have

$$
W=\left\{\begin{array}{c}
\frac{8}{3} b_{j b}^{3}-2 b_{j b}^{2}+2 b_{j b} b_{s b}-b_{s b}^{2}-4 b_{j b}^{2} b_{s b}, \text { if } b_{j b} \geq b_{s b} \\
-\frac{4}{3} b_{s b}^{3}-2 b_{j b}^{2}+2 b_{j b} b_{s b}-b_{s b}^{2}, \text { if } b_{s b}>b_{j b}
\end{array}\right.
$$

Take derivatives of $W$ with respect to $b_{j b}$, we have $b_{j b}^{*}=\frac{b_{s b}}{2}$ if $b_{s b}>b_{j b}$, and $b_{j b}^{*}=b_{s b}$ if $b_{j b} \geq b_{s b}$. Furthermore, $W\left(b_{j b}=\frac{b_{s b}}{2}\right)=-\frac{4}{3} b_{s b}^{3}-\frac{b_{s b}^{2}}{2}>$ $W\left(b_{j b}=b_{s b}\right)$. Therefore, the neutral minister and the conservative senior will jointly select less conservative candidate for junior position.

This is a compromise choice between the preferred choice of the minister and that of the senior. To see this, note that if $b_{s b}>b_{j b}$, then $\frac{\partial E U_{D M}^{H D}}{\partial b_{j b}}=0$ if $b_{j b}=2 b_{s b}^{2}$. Moreover, $E U_{D M}^{H D}\left(b_{j b}=2 b_{s b}^{2}\right)>E U_{D M}^{H D}\left(b_{j b}=b_{s b}\right)$. Therefore the minister prefers to choose the junior with the intensity of conflict as $2 b_{s b}^{2}$. On the other hand, the senior's preferred choice is the junior with $b_{j b}=b_{s b}-2 b_{s b}^{2}$. The analysis above is summarized in the following proposition.

Proposition 3 The minister and the senior has different ideal junior. The junior that maximizes the joint welfare will be in exact middle position between the minister and the senior.

The minister has to tradeoff the efficiency loss from the limitation in low state imposed by the senior, and the gain in more information in high state. When the senior bureaucrat is modest conservative, i.e., $b_{s b} \leq \frac{1}{4}$, then the minister prefers to appoint a junior with the preference close to herself. She knows that though he will impose more restriction on the low state, she gains even more since the like-minded junior can improve efficiency in high state. When the senior bureaucrat is very conservative, i.e., $b_{s b}>\frac{1}{4}$, for the minister the loss due to this extreme senior outweighs the gain in loyalty from the junior, thus she wants to flatter the senior by appointing a junior closely related to him. This logic reverses for the senior. As the result of compromise, these two parties will reach an exactly middle junior.

\subsection{Uncertainty about the preference}

In real world it's usually that the top-level leader doesn't know the true preference of the bottom-level workers due to the limited information processing 
ability or lack of direct access. On the other hand, the middle-level manager may have more precise knowledge about the direct subordinates. This bounded rationality argument provides a rationale for hierarchy (Radner 1993, Bolton and Dewatripont 1994). In our leading example, the minister as an elected politician usually doesn't know the interest of those bottomlevel junior bureaucrats, instead, the senior officer has better idea about the intensity of the conflict of interest of subordinates thanks to the relatively stable organization within a department. In this subsection we explore the delegation scheme in this situation where the knowledge about preference cannot be conveyed to the supervisor. In other words, when the minister designs the job, she just knows the conflict of interest of the senior and the fact that the senior knows the junior.

We assume that the minister only knows $b_{s b}$ and the distribution of $b_{j b}$, $F\left(b_{j b}\right)$. To make the problem tractable, we assume that $b_{j b} \sim U\left[0, \frac{1}{2}\right]$, thus the minister only knows the junior is conservative or liberal, but has no idea about the extent of bias. On the other hand, the senior knows $b_{j b}$ perfectly. Because both agents are of the positive bias, the minister will only choose to ceiling $Y_{s b}$. Using the logic leading to (3), we have that when the junior is more biased:

$E U_{D M}(\bar{y})=\left\{\begin{array}{c}-b_{j b}^{2}\left(1-2 b_{j b}+2 b_{s b}\right)+\frac{\left(2 b_{s b}-b_{j b}\right)^{3}-b_{j b}^{3}}{3}, \text { if } \frac{1}{2}>b_{j b}>1+2 b_{s b}-\bar{y} \\ -b_{j b}^{2} \bar{y}+\frac{2}{3} b_{j b}^{3}+\frac{1}{3}(1-\bar{y})^{3}, \text { if } \frac{1}{2}>1+2 b_{s b}-\bar{y}>b_{j b} \geq b_{s b} \\ -\frac{(1-\bar{y})^{3}}{3}-b_{j b}^{2}\left(\bar{y}-2 b_{s b}+b_{j b}\right)-\frac{\left(2 b_{s b}-b_{j b}\right)^{3}}{3}, \text { if } b_{s b}>b_{j b}>0\end{array}\right.$

Similarly as the intuition underlying Corollary 6 and Proposition 7, when the junior is more biased than the senior, he prefers to give the junior more freedom of action than the minister. Thus if his best ceiling is lower than $\bar{y}$, in other words, the junior's bias is far away from the senior, he can completely implement his preferred delegation set by choosing the upper bound $1+2 b_{s b}-$ $b_{j b}$, as the first row in (9) shows. Otherwise, if his preferred ceiling is higher than $\bar{y}$, which means the junior is aligned to him, the restriction imposed by the minister is binding and the implemented delegation set will also end up in $\bar{y}$, as the second row in (9) suggests. Moreover, if the junior is less biased than him, then he always wants to impose the additional lower bound on the span of control to the junior, as the third row suggests.

The minister gains from the ceiling if the junior is more biased than the senior, since it restricts the senior's tendency to relegate more freedom in high state. However, the tight cap precludes possible beneficial adaptation in high state when the junior is like-minded. The optimal delegation scheme 
balances loss of control and gain in knowledge, albeit now the information is the knowledge about the intensity of preference misalignment. The precise ceiling depends on which scenario above is more likely to occur. Furthermore, the like-minded senior enables her to have better control of the informed junior. In the limit case that $b_{s b}=0$, the senior is perfectly aligned to the minister and she can relegate him full control of action. We calculate the expected payoff to the minister under the uncertainty about the junior's bias, and found out that it's optimal for her to grant complete delegation set when the senior is not too biased ${ }^{11}$. Formally:

Proposition 4 If the minister has uniform prior about the bias of the junior over the support $\left[0, \frac{1}{2}\right]$, then:

1. It's optimal to her to set $\bar{y}$ such as

$$
\bar{y}=\left\{\begin{array}{c}
1, \text { if } b_{s b}<\frac{1}{4} \\
1-\frac{\sqrt{3}}{6}, \text { if } b_{s b}>\frac{1}{4}
\end{array}\right.
$$

2. If the ignorant minister can directly delegate to the junior, she will choose $\bar{y}=1-\frac{\sqrt{3}}{6}$.

3. For large span of the bias of the senior, it's optimal for the minister to delegate to the senior, instead of delegating to the junior even if she has the opportunity.

The part 1) says that the upper bound of the delegation set varies with respect to the bias of the senior, but not continuously. The part 2) says that if the minister is uncertain about the preferences of the informed agent, she will give him less discretion. This is consistent with the insights of Armstrong (1995). The part 3) compares direct delegation with indirect delegation thorough a strategic senior, and demonstrates that delegating to a knowledgeable biased senior may benefit the minister more than direct delegation.

This result provides a rationale for the exclusive concentration on full delegation among researchers (Dessein 2002, Ambrus et al 2009): when the minister is uncertain about the conflict of interest of the informed party, and the discrepancy of interest between the senior and her is not too large, it will be beneficial to delegate all decision right to the senior who has the relevant knowledge about the intensity of conflict. The complete delegation scheme thus arises as the optimization outcome of the minister.

\footnotetext{
${ }^{11}$ The quantitative result is obtained by the specification of the prior distribution. However, the qualitative results still remain.
} 
Moreover, this provides an additional rationale for the emergence of hierarchy. We compare the expected payoff to the minister if she can delegate to the junior directly, though she still doesn't know the exact bias of the junior. It's shown that if the conflict of interest of the senior is not too large $\left(b_{s b}<\frac{1}{5}\right)$, then delegating to an informed senior will generate higher expected payoff to the minister than direct delegation. A slightly biased knowledgeable senior provides the minister with the better control of the informed party, thus the hierarchical structure itself exemplifies the tradeoff between the loss of control to the senior and gain in the knowledge of the senior. The minister optimally forgoes the direct access to the unacquainted informed party, and relies on the acquainted knowledgeable intermediary to control the bottom executives instead. The asymmetric knowledge between the senior and the minister thus leads not only to complete delegation, but also the use of hierarchy to control informed agents.

It worth noting that the minister cannot gain by adding a pre-play communication stage in which the agents can send a report about $b_{j b} .{ }^{12}$ That's because the optimal delegation scheme prescribes that the minister only imposes a ceiling on $Y_{s b}$, which is determined by $b_{j b}$. Therefore, both the junior and the senior have the common interest to convince the minister that there is no conflict of interest of the junior, i.e., $b_{j b}=0$. Thus both reports are uninformative and the preplay communication leads babbling equilibrium. If the minister announces that the junior can be promoted if the reported $b_{j b}<b_{s b}$, this promise is not credible since by Corollary 11, it's not always in the interest of the minister to assign the more loyal agent to the senior position.

\section{Conclusion}

The fact that within hierarchical organization, especially government, the span of discretionary authority decreases top down motivates our research on optimal interval delegation in hierarchy. This chapter contributes to literature by providing a complete characterization of the implemented interval delegation set, and establishing conditions for attaining efficiency in hierarchy. Moreover, we also endogenize the hierarchy structure and complete delegation as response to incomplete knowledge about the conflict of interest. The results that the optimal intermediary in delegation chain should be moderately biased, and allowing transfer of authority benefits decision maker are intuitive. We also use this framework to show that the HRM practices within

\footnotetext{
${ }^{12}$ The author thanks David Martimort for raising this point.
} 
bureaucratic organization, i.e., job design, promotion, and hiring policy, are interrelated.

It worthwhile to note that in most parts we study the three-tier hierarchy and ignore the multiple subordinates tree structure, which is a more realistic feature of hierarchy. However, as long as hierarchy is formed based on the consideration beyond strategic information transmission, such as information processing cost (Radner, 1993), heterogenous knowledge (Garicano, 2000) or conflict over hiring and promotion decisions (Friebel and Raith, 2004), our results still hold in multiple subordination structure.

Mechanism design literature has demonstrated that the principal could not be worse off by commitment. However, this paper shows that given hierarchical structure and divergence of interest, the set of decision rule available to the minister is limited by the incentive of mediator, thus the minister may find it's no longer optimal to make a specific commitment: delegation. This result suggests that network structure matters in mechanism design and implementation. Delegation is usually considered as an important form of incomplete contract, which works as response to ex post renegotiation or unverifiable information. This chapter shows that specific network structure can impose stringent restriction on the efficiency of incomplete contract, which is to a large extent still beyond the sights of theorists.

In this paper we stress interval delegation, which is in widespread use and realistic in our budget approval example. However, for opposite biased or more biased senior, interval delegation may not be the optimal delegation scheme for the minister. Actually the optimal delegation may take the form of an interval plus a discrete point. However, this optimal scheme though may be of theoretical interest, is not realistic in bureaucratic situation, especially in budget planning procedure in which free disposition may preclude any possibility of discrete $\operatorname{action}^{13}$.

\section{A Appendix A}

Proof of Lemma 1: See Lemma 1 and Theorem 1 in CS.

Proof of Lemma 2: See Theorem 1 in GHPS (2009).

Proof of Lemma 3: Proposition 2.1 in AM (2008) establishes the conditions for interval delegation. They define backward bias $T(\theta)$ (see (1) there). In quardratic-utility uniform context they are $T(\theta)=\theta\left(\frac{\theta}{2}+b\right)$, which is strictly convex for all $\theta$. Thus the optimal delegation set is a connected set.

\section{Proof of Corollary 1}

\footnotetext{
${ }^{13}$ The author thanks Oliver Hart for suggesting this point.
} 
By Lemma 3 the delegation set is a connected set. Without loss of generality, let this connected set be a closed interval of state $\left[\min \left\{\underline{\theta}+b_{j b}, 0\right\}, \max \left\{\bar{\theta}+b_{j b}, 1\right\}\right] \in$ $Y$ where the informed party could select any action within this interval, i.e., the informed party can implement his preferred action when $\theta \in[\underline{\theta}, \bar{\theta}]$. For any $\bar{\theta}+b_{j b} \leq 1$ to be optimal from the minister standpoint, we need that she is indifferent between her best response given that $\theta \geq \bar{\theta}$ and delegated action $\bar{\theta}+b_{j b}$. This would lead to $1-2 b_{j b}=\bar{\theta}$. Since $b_{j b}<0$, this would be impossible, so $\bar{\theta}=1$ has to be satisfied.

On the other hand, this the lower bound $\underline{\theta}+b_{j b}$ should also be the best response of the minister when she knows that the true state locates within $[0, \underline{\theta}]$, thus this $\underline{\theta}$ should be equal to $-2 b_{j b}$. Therefore we reach the desired conclusion.

\section{Proof of Proposition 1}

Since $Y_{j b} \subset Y_{s b}$, the highest action available to the junior $\overline{y_{j b}}$ can not exceed the highest action in $Y_{s b}$. On the other hand, by Lemma 2 if the senior could select whatever delegation set in his interest, then the size of delegation set relies only on the relative difference of bias $\Delta$, thus the upperbound would be $1-\Delta$.

\section{Proof of Corollary 2}

Lemma 3 implies that $Y_{j b}$ is connected set if $Y_{s b}$ is connected, thus the the minister can focus only on the upper-bound. Since $\left.\Delta<b_{j b}, 1-\Delta\right\rangle$ $\sup \left\{y \mid y \in Y_{s b}\right\}=1-b_{j b}$, thus by truncating delegation set to the senior with the highest action of efficient delegation set $1-b_{j b}$, the junior would be limited to take any action less than $1-b_{j b}$, which is exactly the contents of efficient delegation set.

\section{Proof of Proposition 2}

1. We first study the case of opposite biased agents and show that the efficient delegation set is not implementable. Without loss of generality, assume that $b_{j b}>0>b_{s b}$, so $\Delta>b_{j b}>0$. Suppose in contrast, the efficient delegation set $Y^{*}=\left[0,1-b_{j b}\right]$ is implementable, then we should have $Y^{*} \subset Y_{s b}$, namely there is an interval larger than $\left[0,1-b_{j b}\right]$ belongs to the senior's delegation set.

Because the relative difference of interest is $\Delta$ and $1-\Delta<1-b_{j b}=$ $\sup \left\{y \mid y \in Y^{*}\right\}$, by Proposition 1 the senior would like to only delegate $[0,1-\Delta]$ to the junior, given his delegation set $Y_{s b}$. Hence we get the contradiction needed.

2. Now we check the case of extremely biased senior. Still without loss of generality, we assume that $b_{s b}>b_{j b}>0$. Hence $\Delta<0$, and according to Corollary 1 , the senior would impose lower bound $-\Delta$ on $Y_{j b}$, while 
$Y^{*}$ prescribes zero lower bound. Since the senior' prefers to truncate at the bottom, the minister could not implement the efficient delegation set, in which the junior has control in low state.

Proof of Lemma 4: See Ivanov (2009) Theorem 1 and Lemma 4.

\section{Proof of Corollary 3}

If the officer 2 is assigned as the junior, then as (4) shows, the expected payoff to the minister is $\left(\frac{4}{3} b_{2}-1\right) b_{2}^{2}$, while if the officer 1 becomes the junior the expected payoff becomes $-\frac{8}{3} b_{2}^{3}+4 b_{1} b_{2}^{2}-b_{1}^{2}$. By comparing these two formula it shows that it's beneficial to appoint officer 2 only if $4 b_{2}^{3}-\left(1+4 b_{1}\right) b_{2}^{2}+b_{1}^{2}>0$. The remaining reasonable root leads to $b_{2}>\frac{1+\sqrt{1+16 b_{1}}}{8}$.

\section{Proof of Proposition 3}

By (9), we divide the discussion into two parts: the binding ceiling level $\bar{y}$ or not.

1. First, we study the case that the upper bound is always binding, namely $\bar{y}<1-b_{j b}+2 b_{s b}$ whenever $b_{s b}<b_{j b}<\frac{1}{2}$. This condition translates into $\bar{y}<\frac{1}{2}+2 b_{s b}$. Thus we would use only the second and third row of (9)

Therefore, the ex ante expected payoff to the minister is

$$
\begin{aligned}
& \int_{0}^{b_{s b}}\left[\frac{(\bar{y}-1)^{3}}{3}-b_{j b}^{2}\left(\bar{y}-2 b_{s b}+b_{j b}\right)-\frac{\left(2 b_{s b}-b_{j b}\right)^{3}}{3}\right] d F\left(b_{j b}\right)(10) \\
& \int_{b_{s b}}^{\frac{1}{2}}\left[\frac{(\bar{y}-1)^{3}}{3}-b_{j b}^{2} \bar{y}+\frac{2}{3} b_{j b}^{3}\right] d F\left(b_{j b}\right)
\end{aligned}
$$

The first term is the expected payoff if the junior is less biased than the senior, where the senior will set the lower bound on $Y_{j b}$. The second term represents the expected payoff if the junior is more biased than the senior, where the senior will further delegate the control rights to the junior up till $\bar{y}$, the cap of his own authority.

Take the derivative of the above (10) and equal it to zero, we have $\bar{y}=1-\left[\int_{0}^{\frac{1}{2}} b_{j b}^{2} d F\left(b_{j b}\right)\right]^{\frac{1}{2}}$. Using the fact that $F$ is uniform distribution we have $\bar{y}=1-\frac{\sqrt{3}}{6}$ and

$$
E U(\bar{y})=-\frac{1}{16}+\frac{\sqrt{3}}{108}-2 b_{s b}^{4}
$$


Check it with the initial condition $\bar{y}<\frac{1}{2}+b_{s b}$, we need $b_{s b} \in\left[\frac{1}{4}-\frac{\sqrt{3}}{12}, \frac{1}{2}\right]$.

2. Second, we study the case that the cap sometimes may not hold, in other words, $\bar{y}>\frac{1}{2}+b_{s b}$, thus the senior may aslo imposes his preferred ceiling. Now all the three rows in (9) will enter the expected payoff to the minister, which can be expressed as

$$
\begin{aligned}
& \int_{0}^{b_{s b}}\left[\frac{(\bar{y}-1)^{3}}{3}-b_{j b}^{2}\left(\bar{y}-2 b_{s b}+b_{j b}\right)-\frac{\left(2 b_{s b}-b_{j b}\right)^{3}}{3}\right] d F\left(b_{j b}\right)+ \\
& \int_{b_{s b}}^{1-\bar{y}+2 b_{s b}}\left[\frac{(\bar{y}-1)^{3}}{3}-b_{j b}^{2} \bar{y}+\frac{2}{3} b_{j b}^{3}\right] d F\left(b_{j b}\right)+ \\
& \int_{1+2 b_{s b}-\bar{y}}^{\frac{1}{2}}\left[\frac{\left(2 b_{s b}-b_{j b}\right)^{3}+b_{j b}^{3}}{3}-b_{j b}^{2}\left(1+2 b_{s b}-2 b_{j b}\right)\right] d F\left(b_{j b}\right)
\end{aligned}
$$

The first term is the same as that in (10). The second term establishes that when the junior's preference is not far from the senior, the cap $\bar{y}$ binds $Y_{j b}$ since the senior always prefer to delegate more to the junior. The third term shows that when the junior is farther from the senior, the senior will impose the ceiling in his interest.

The first order condition suggests that $\bar{y}=1+2 b_{s b}$ or $1+b_{s b}$, both are impossible since the highest action in $Y$ is 1 . However, since the second order condition is always negative, we know that the minister always gains by setting a higher $\bar{y}$. Hence the minister will choose the highest possible ceiling in his action set $Y$, the optimal $\bar{y}=1$. In other words, the minister will completely delegate to the senior. The expected payoff is

$$
E U(\bar{y})=-\frac{1}{24}-3 b_{s b}^{4}
$$

We return to check the initial condition and get $b_{s b}<\frac{1}{4}$.

3. Finally, since the results in step 1 and 2 has overlap when $b_{s b} \in$ $\left[\frac{1}{4}-\frac{\sqrt{3}}{12}, \frac{1}{4}\right]$, we compare the expected payoff to the minister under these two $\bar{y}$ and find out that $\bar{y}=1$ generates higher payoff.

Thus we finish the first part of this proposition. Now we turn to the second part. Now the minister can directly delegate to the junior, but she still has no knowledge about the bias of the junior. First, we have that for a given upper bound $\bar{y}$, the expected payoff to the minister is 


$$
E U_{D M}(\bar{y})=-\int_{0}^{\bar{y}-b_{j b}} b_{j b}^{2} d \theta-\int_{\bar{y}-b_{j b}}^{1}(\bar{y}-\theta)^{2} d \theta=-b_{j b}^{2} \bar{y}+\frac{2}{3} b_{j b}^{3}+\frac{(\bar{y}-1)^{3}}{3}
$$

Then the minister will choose $\bar{y}$ to maximize $\int_{0}^{\frac{1}{2}} E U_{D M}(\bar{y}) d F\left(b_{j b}\right)$, the first order condition turns out to be $\bar{y}=1-\left[\int_{0}^{\frac{1}{2}} b_{j b}^{2} d F\left(b_{j b}\right)\right]^{\frac{1}{2}}=1-\frac{\sqrt{3}}{6}$. Thus we get the expected payoff under the optimal direct delegation scheme is

$$
E U(\bar{y})=-\frac{1}{16}+\frac{\sqrt{3}}{108}
$$

It's easy to see that (12) is always smaller than (14), thus we concentrate on comparing (13) and (14). Then we have whenever $b_{s b} \leq\left(\frac{1}{144}-\frac{\sqrt{3}}{324}\right)^{\frac{1}{4}} \approx$ $0.1999,(13)$ is larger than (14). In other words, delegating to an informed senior dominates delegating to the junior directly.

\section{B Appendix B}

We follow Ambrus et al (2009) closely to construct the two-action mixed strategy equilibrium in hierarchical communication. We focus on the outcomeequivalent perfect Bayesian-Nash equilibrium that the junior reports $s_{1}$ at any $\theta \in[0, x]$ and $s_{2}$ at any $\theta \in(x, 1]$, the negatively biased senior $\left(b_{s b}<0\right)$ recommends action $m_{1}$ to the minister after receiving message $s_{1}$, and mixes between $m_{1}$ (with probability $p$ ) and $m_{2}$ after getting message $s_{2}$. Finally the minister acts according to the report sent by the senior, $y_{1}=m_{1}$ and $y_{2}=m_{2}$.

Since the minister understands for sure that $\theta \geq x$ when she hears $m_{2}$, we need $m_{2}=\frac{1+x}{2}$. Because the senior must be indifferent between sending $m_{1}$ and $m_{2}$ when he receives $s_{1}$, we have $m_{1}=\frac{1+x}{2}+2 b_{s b}$. Similarly, the junior has to be indifferent between both actions at $\theta=x$, so we get $x=1-2 \Delta$. Thus we substitute it into $m_{1}$ and $m_{2}$, and solve $m_{2}=1-\Delta$ and $m_{1}=1-\Delta+2 b_{s b}$. By Bayesian Law, the minister must update her posterior in such way that $E\left(\theta \mid m_{1}\right)=m_{1}$, so we have

$$
\frac{x}{2} \operatorname{Pr}\left(\theta \in[0, x] \mid m_{1}\right)+\frac{1+x}{2} \operatorname{Pr}\left(\theta \in[x, 1] \mid m_{2}\right)=m_{1}
$$

where $\operatorname{Pr}\left(\theta \in[0, x] \mid m_{1}\right)=\frac{x}{x+(1-x) p}$

Thus we get $p=-\frac{1}{8} \frac{(1-2 \Delta)\left(1+4 b_{s b}\right)}{\Delta b_{s b}}$.

By the feasibility requirement that $p \in[0,1]$ we get $0 \leq \Delta \leq \frac{1}{2}, b_{s b} \geq-\frac{1}{4}$, and $\Delta \geq \frac{1}{2}\left(1+4 b_{s b}\right)$. 


\section{References}

[1] Alonso, Ricardo and Niko Matouschek (2008) Optimal Delegation, Review of Economic Studies, 75, 259-293.

[2] Ambrus, Attila.; Eduardo Azevedo. and Yuichiro Kamada (2009) Hierarchical Cheap Talk, Working Paper, Harvard University.

[3] Ambrus, Attila.; Eduardo Azevedo.; Yuichiro Kamada. and Yuki Takagi (2010) Legislative Committees as Information Intermediaries: a Unified Theory of Committee Selection and Amendment Rules, Working Paper, Harvard University.

[4] Armstrong, Mark (1995) Delegation and Discretion, Working Paper, University College London.

[5] Blume, Andreas.; Oliver J. Board and Kohei Kawamura (2007) Noisy Talk, Theoretical Economics, 2, 395-440.

[6] Bolton, Patrick and Mathias Dewatripont (1994) The Firm as a Communication Network, Quarterly Journal of Economics, 109, 809-839.

[7] Calvo-Armengol, Antoni.; Joan de Marti Beltran and Andrea Prat (2009) Endogenous Communication in Complex Organizations, Working Paper, Universitat Pompeu Fabra and LSE.

[8] Crawford, Vincent and Joel Sobel (1982) Strategic Information Transmission, Econometrica, 50, 1431-1451.

[9] Dessein, Wouter (2002) Authority and Communication in Organizations, Review of Economic Studies, 69, 811-838.

[10] Friebel,Guido. and Michael Raith (2004) Abuse of Authority and Hierarchical Communication, RAND Journal of Economics, 35, 224-244.

[11] Garicano, Luis (2000) Hierarchies and the Organization of Knowledge in Production, Journal of Political Economy, 108, 874-904

[12] Geanakoplos, John and Paul Milgrom (1991) A Theory of Hierarchies Based on Limited Managerial Attention, Journal of the Japanese and International Economies, 5, 205-225.

[13] Goltsman, Maria.; Johannes Horner.; Gregory Pavlov and Francesco Squintani (2009) Mediation, Arbitration and Negotiation, Journal of Economic Theory, 144, 1397-1420. 
[14] Gibbons, Robert and M. Waldman (1999) Careers in Organization: Theory and Evidence, in Handbook of Labor Economics, ed. O. Ashenfelter and D. Card, North Holland.

[15] Holmstrom, Bengt (1977) On Incentives and Control in Organization, Ph.D. Dissertation, Graduate School of Business, Stanford University.

[16] Ivanov, Maxim (2009) Communication via a Strategic Mediator, Journal of Economic Theory, 145, 869-884.

[17] Iyer, Lakshmi and Anandi Mani (2009) Traveling Agents: Political Change and Bureaucratic Turnover in India, Working Paper, Harvard Business School.

[18] Kovac, Eugen and Tymofiy Mylovanov (2009) Stochastic Mechanisms in Settings without Monetary Transfers: The Regular Case, Journal of Economic Theory, 144, 1373-1395.

[19] Melumad, Nahum D and Toshiyuki Shibano (1991) Communication in Settings with No Transfers, RAND Journal of Economics, 22, 173-198.

[20] Mitusch, Kay. and Roland Strausz (2005) Mediation in Situations of Conflict and Limited Commitment, Journal of Law, Economics, and Organization, 21, 467-500.

[21] Myerson, Roger (1982) Optimal Coordination Mechanisms in Generalized Principal-agent Problems, Journal of Mathematical Economics, 10, $67-81$.

[22] (1986) Mltistage Games with Communication, Econometrica, 54, 323-358.

[23] Mylovanov, Timofiy (2008) Veto-Based Delegation, Journal of Economic Theory, 138, 297-307.

[24] Radner, Roy (1993) The Organization of Decentralized Information Processing, Econometrica, 61, 1109-1146.

[25] Renou, Ludovic and Tristan Tomala (2008) Mechanism Design and Communication Networks, Working Paper, Leicester University.

[26] Tirole, Jean (1986) Hierarchies and Bureaucracies: On the Role of Collusion in Organizations, Journal of Law, Economics, and Organization, $2,181-214$. 
[27] Warren, Patrick (2008) Allies and Adversaries: How Appointees Drive Policymaking under Separation of Powers, Working Paper, Clemson University.

[28] Weber, Max (1946) Bureaucracy, in From Max Weber: Essays in Sociology, ed. H. Gerth and C. W. Mills, New York: Oxford University Press. 